\title{
Metodo di calcolo della magnitudo di terremoti registrati da sismografi in uso agli inizi del nostro secolo
}

(Calculation method of magnitude of earthquakes recorded by seismographs used at the beginning of our century)

\author{
G. Casal - R. Console $(*)-$ B. De Simoni $(*)$
}

Received on October 28 th, 1977

Riassunto. - Si espone un metodo di calcolo per poter determinare la magnitudo mediante la registrazione di sismografi in uso agli inizi del secolo. Si perviene ad una espressione generalizzata della magnitudo valida per strumenti che hanno il coefficiente di smorzamento $h$ dell'ordine di 0.01 , con periodi compresi tra 1.2 e $6.7 \mathrm{sec}$, per distanze epicentrali fino a $600 \mathrm{~km}$. I risultati ottenuti hanno messo in evidenza la validità del metodo.

Summary. - A method of calculation to determine the magnitude by means of the records of seismographs used at beginning of the century is exposed. One can obtain an expression generalized of magnitude good for seismographs, which have the damping coefficient $h$ of range 0.01 about, with periods from 1.2 to 6.7 seconds inclusive, and for the epicentral distance as far as $600 \mathrm{~km}$. The results have shown the validity of the method.

\section{Premessa.}

La magnitudo per terremoti locali fu definita da Richter ( $\left.{ }^{4}\right)$ dalla relazione numerica:

$$
M=\log \frac{A_{N S}+A_{E W}}{2}+f(\Delta)
$$

$\left(^{*}\right)$ Istituto Nazionale di Geofisica - Oss. Geofis. Centrale - Monte Porzic, Catone (Roma). 
Le grandezze $A_{N S}, A_{E W}$ rappresentanti le ampiezze massime in $\mathrm{mm}$ sulle due componenti $N S$ ed $E W$ si determinano dall'analisi delle registrazioni della coppia campione Wood-Anderson, con le caratteristiche $T_{0}=0.8 \mathrm{sec}$., $V_{0}=2800, h_{0}=0.8$.

Il termine $f(\Delta)$ è un termine correttivo dipendente dalla distanza epicentrale.

E stato mostrato $\left({ }^{1}\right)$ come sia possibile ottenere la magnitudo da registrazioni di altre coppie di sismografi appartenenti alla classe degli strumenti a breve periodo, ridefinendo per essi opportunamente la funzione $f(\Delta)$.

Il modo di procedere è sperimentale avendo posto in funzione nello stesso luogo sia il sismografo campione che un secondo strumento di caratteristiche arbitrarie; sarà possibile procedere ad una taratura soltanto dopo un numero assai grande di registrazioni di eventi sismici con distanze epicentrali diverse.

Lo scopo del presente lavoro è finalizzato al recupero e ad un'ulteriore analisi di una considerevole quantità di dati di terremoti registrati da sismografi antichi, i quali, come è noto, hanno costanti strumentali molto diverse.

Per ovvie ragioni la taratura diretta non è stata possibile; si è utilizzato, quindi, un metodo numerico tramite l'utilizzazione di un elaboratore elettronico.

\section{Correlazione fra REgISTRAZIONI di STRUMENTI DIVERSi.}

Scelte quattro registrazioni di terremoti a opportune distanze epicentrali, effettuate con la coppia campione, esse sono state digitalizzate, e i dati così ottenuti sono stati introdotti al posto di $x(t)$ nella seguente equazione differenziale

$$
G_{0} \ddot{x}(t)+W_{0} \dot{x}(t)+Q_{0} x(t)=-f(t)
$$

dove si è posto

$$
G_{0}=\frac{1}{V_{0}} ; \quad W_{\mathrm{v}}=\frac{4 \pi h_{0}}{V_{0} T_{0}} ; \quad \hat{\mathrm{v} v}=\frac{4 \pi^{2}}{V_{\mathrm{1}} T^{2}} .
$$

La soluzione fornisce l'accelerazione del terreno $f(t)$, conoscendo la quale si è potuta costruire la registrazione che si sarebbe ottenuta da 
un qualsivoglia sismografo, governato da un'equazione di tipo [2], introducendo le opportune costanti.

La soluzione della [2] è stata eseguita con il calcolatore ipotizzando un andamento lineare tra i punti della $f(t)$.

1 risultati ottenuti su alcuni casi scelti per prova, sono stati soddisfacenti. Essi sono stati controllati con una verifica la cui logica è riportata nello schema a blocchi di fig. 1 .

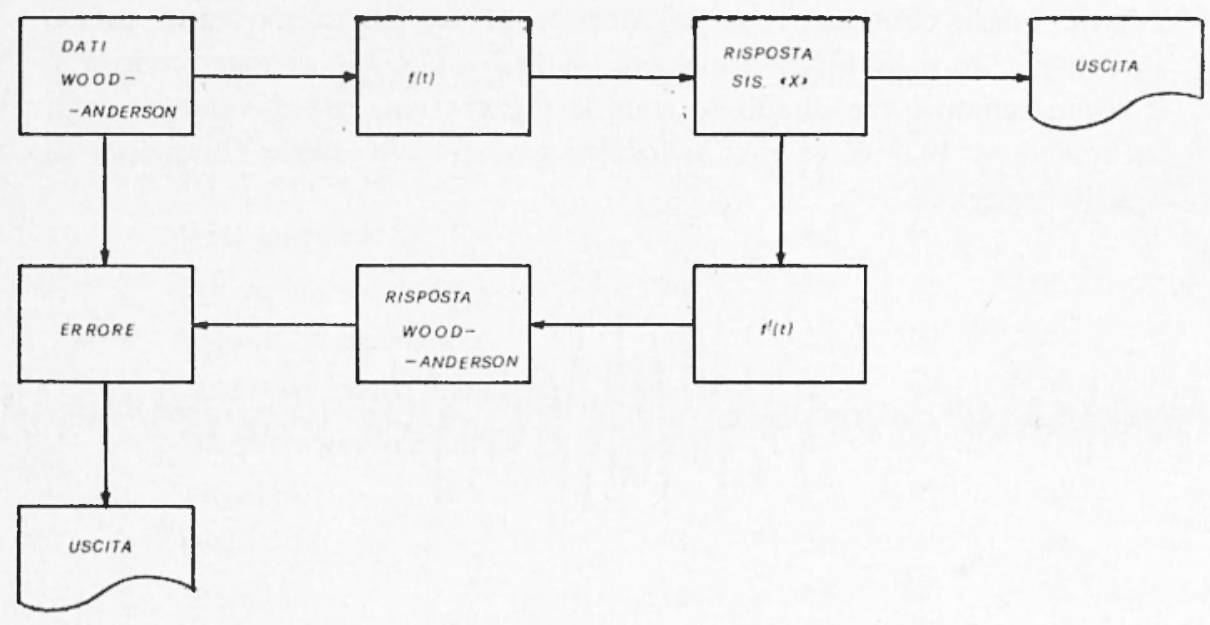

Fig. 1 - Schema a blocchi del programma adoperato nel controllo del metodo per correlare registrazioni di differenti sismografi.

\section{Alcune considerazioni riguardanti gli antichi sismografi.}

Le caratteristiche strumentali di alcuni sismografi in uso all'inizio del secolo sono riportate in Tab. 1; esse sono state tratte dai Bollettini della Società Sismologica Italiana.

La difficoltà maggiore riscontrata sta nella valutazione del coefficiente di smorzamento $h_{x}$, il quale, secondo la logica costruttiva dell'epoca, si riteneva dovesse essere il più piccolo possibile. Esso era provocato esclusivamente dai diversi inevitabili attriti delle parti costituenti lo strumento.

Dall'analisi di alcuni sismografi è stata possibile la valutazione di $h_{x}$ in fase di oscillazioni pendolari libere; il valore ottenuto è compreso 
tra $10^{-2}$ e $10^{-3}$. Sono state effettuate diverse prove numeriche onde valutare l'influenza della imperfetta conoscenza del valore di $h_{x}$ sui risultati.

Tali prove ci hanno condotto a ritenere non rilevante l'esatta valutazione di questo parametro e quindi si è deciso di fissare per $h_{x}$ il valore di 0.01 , onde semplificare procedimenti numerici ed analitici.

A titolo dimostrativo riportiamo in fig. 2a una registrazione effettuata mediante la coppia campione, sita nell'Osservatorio Centrale di Monte Porzio Catone. Essa si riferisce al terremoto avvenuto presso Ascoli Piceno il 24.11.1972 alle ore 08.06.26 (TGM), di magnitudo 3.8. I sismogrammi $b$ e $c$ sarebbero state le registrazioni rispettivamente degli strumenti n. 19 e 6 , se essi si fossero trovati nelle stesse condizioni di quello campione.
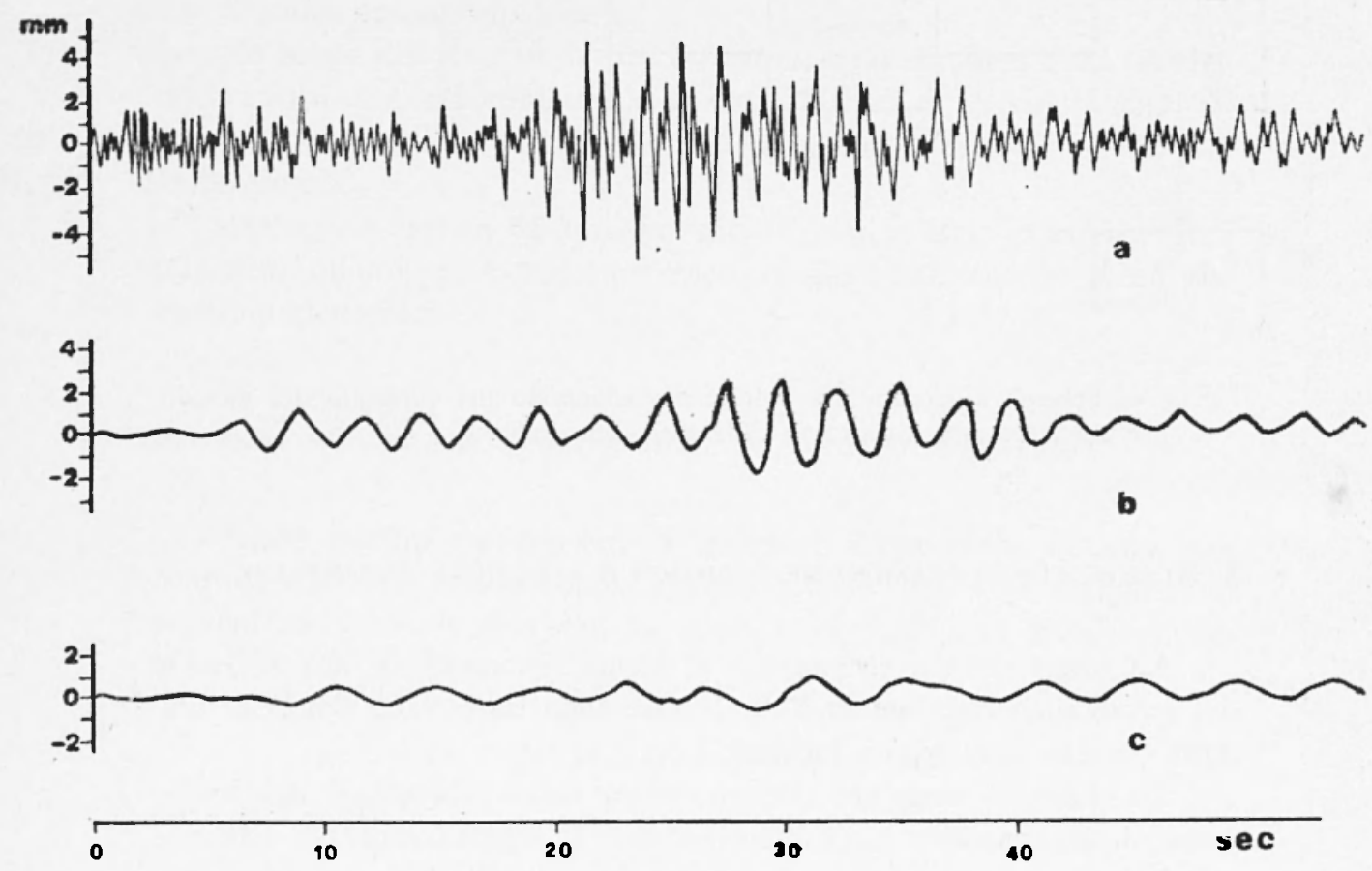

Fig. 2 - Confronto fra la registrazione ottenuta con il sismografo Wood-Anderson e quelle ricavate analiticamente per gli strumenti riferiti nel testo con il numero 19 e 6 . Il terremoto è quello di Ascoli Piceno del 24.11.72, $08^{\prime \prime} 06^{\prime \prime} 26^{5}$, $\operatorname{con} M_{:}=3.8$ c $A=126 \mathrm{~km}$. 
TABELLA 1

Caratteristiche strumentali.

\begin{tabular}{|c|c|c|c|c|c|}
\hline n. & $\begin{array}{l}\text { Denominazione dello } \\
\text { strumento }\end{array}$ & $\begin{array}{c}\text { Massa } \\
(\mathrm{kg})\end{array}$ & $\begin{array}{l}\text { Periodo } \\
\text { sec. }\end{array}$ & $\begin{array}{l}\text { Ingran. } \\
\text { statico }\end{array}$ & $\begin{array}{l}\text { Vel. scorr. } \\
(\mathrm{cm} / \mathrm{h})\end{array}$ \\
\hline 1 & Grande $S G$ Cancani & 300 & 10.0 & 12.5 & 60 \\
\hline 2 & $M S G$ Vicentini & 100 & 2.4 & 70 & 60 \\
\hline 3 & Nuovo $S G$ Agamennone & 80 & 2.6 & 100 & 60 \\
\hline 4 & Vicentini & 106 & 2.4 & 100 & 30 \\
\hline 5 & SG Agamennone & 112 & 5.0 & 12 & 30 \\
\hline 6 & $M S G$ Agamennone & 500 & 4.2 & 60 & 70 \\
\hline 7 & $S G$ Agamennone & 200 & 4.4 & 14 & 35 \\
\hline 8 & Vicentini a pantografo & 450 & 2.4 & 100 & 100 \\
\hline 9 & $M S G$ Vicentini a pantografo & 500 & 4.6 & 80 & 180 \\
\hline 10 & Microsismometrografo « $B »$ & 100 & 2.3 & 128 & 51 \\
\hline 11 & Vicentini & 50 & 2.3 & 85 & 10 \\
\hline 12 & Vicentini & 66.5 & 2.4 & 80 & 20 \\
\hline 13 & Vicentini & 100 & 2.4 & 120 & 30 \\
\hline 14 & Agamennone & 220 & 3.9 & 10 & 34 \\
\hline 15 & Vicentini & 150 & 2.4 & 65 & 30 \\
\hline 16 & $M S$ Vicentini & 100 & 2.4 & 80 & 60 \\
\hline 17 & Agamennone & 200 & 6.0 & 20 & 29 \\
\hline 18 & Nuovo $M S G$ Agamennone & 250 & 2.6 & 100 & 60 \\
\hline 19 & Nuovo MSG Agamennone & 1500 & 2.6 & 160 & 60 \\
\hline 20 & Grande $S M$ & 200 & 3.0 & 20 & 60 \\
\hline 21 & Sis. a lastra affumicata & 26.4 & 3.5 & 10 & 2670 \\
\hline 22 & $M S G$ Vicentini & 100 & 2.3 & 50 & 60 \\
\hline 23 & $S G$ Agamennone & 200 & 6.0 & 12.5 & 36 \\
\hline 24 & Pendolo $N$ Stiattesi & 260 & 8.6 & 25 & 100 \\
\hline 25 & Pendolo $E$ Stiattesi & 260 & 8.0 & 25 & 100 \\
\hline
\end{tabular}




\section{Espressione generalizzata della magnitudo.}

La magnitudo calcolata mediante un qualsiasi strumento $x$ deve essere funzione, oltre che delle caratteristiche strumentali $\left(V_{x}, T_{x}, h_{x}\right)$, della distanza epicentrale; ne diamo quindi la seguente espressione:

$$
M=\log A_{X M}+f(\Delta)+g\left(V_{x}, T_{x}, h_{x}, \Delta\right)
$$

dove $A_{X M}$ è la massima ampiezza registrata dal sismografo che si vuole tarare. Secondo numerose verifiche eseguite su registrazioni del WoodAnderson, le ampiezze massime sulle due componenti sono comparabili tra loro; ciò permette di semplificare la [1] la quale assume la seguente forma:

$$
M=\log A_{W A M}+f(\Delta)
$$

dove $A_{W A M}$ è la massima ampiezza per una delle due componenti. Uguagliando la [3] alla [1']

$$
\log \frac{A_{W A 1}}{A_{X M}}=g\left(V_{x}, T_{x}, h_{x}, \Delta\right)
$$

Indicando con $A_{x u}$ l'ampiezza del moto della massa oscillante, si ha: $A_{X M}=V_{x} A_{X U}$, quindi

$$
\log \frac{A_{W A M}}{A_{X U}}=g\left(T_{x}, \Delta\right) .
$$

Si è giunti ad una funzione dipendente soltanto da $T_{x}$ e $\Delta ; h_{x}$, infattì, è stato precedentemente fissato e la dipendenza dell'amplificazione statica eliminata, e normalizzata ad uno.

La funzione di correzione $g\left(T_{x}, \Delta\right)$ è stata calcolata con il metodo descritto nel secondo paragrafo, per ciascuno dei quattro terremoti effettivi in funzione di $\Delta$ e del periodo $T_{x}$.

I risultati ottenuti sono riportati in Tab. 2. Mediante una regressione lineare dei dati si è potuto scrivere per ogni fissato periodo:

$$
g\left(T_{x}, \Delta\right)=a\left(T_{x}\right) \Delta+b\left(T_{x}\right) .
$$

In base ai gruppi di dati sperimentali, $a\left(T_{x}\right)$ e $b\left(T_{x}\right)$ hanno dipen- 
denza lineare dal periodo, scriviamo perciò:

$$
\begin{aligned}
& a\left(T_{x}\right)=k T_{x}+l \\
& b\left(T_{x}\right)=p T_{x}+q .
\end{aligned}
$$

Sostituendo nella $g\left(T_{x}, \Delta\right)$ diviene:

$$
g\left(T_{x}, \Delta\right)=k \Delta T_{x}+l \Delta+p T_{\mathfrak{r}}+q
$$

e con calcolo esplicito

$$
g\left(T_{x}, \Delta\right)=\left(0.65 \Delta T_{x}-21 \Delta-1729 T_{s}\right) 10^{-4}+3.4273 .
$$

In fig. 3 è riportata una famiglia di curve $g\left(T_{x}, \Delta\right)$.

Arriviamo, quindi, alla formula desiderata:

$$
M=\log A_{X M}-\log V_{x}+g\left(T_{\mathfrak{r}}, \Delta\right)+f(\Delta)
$$

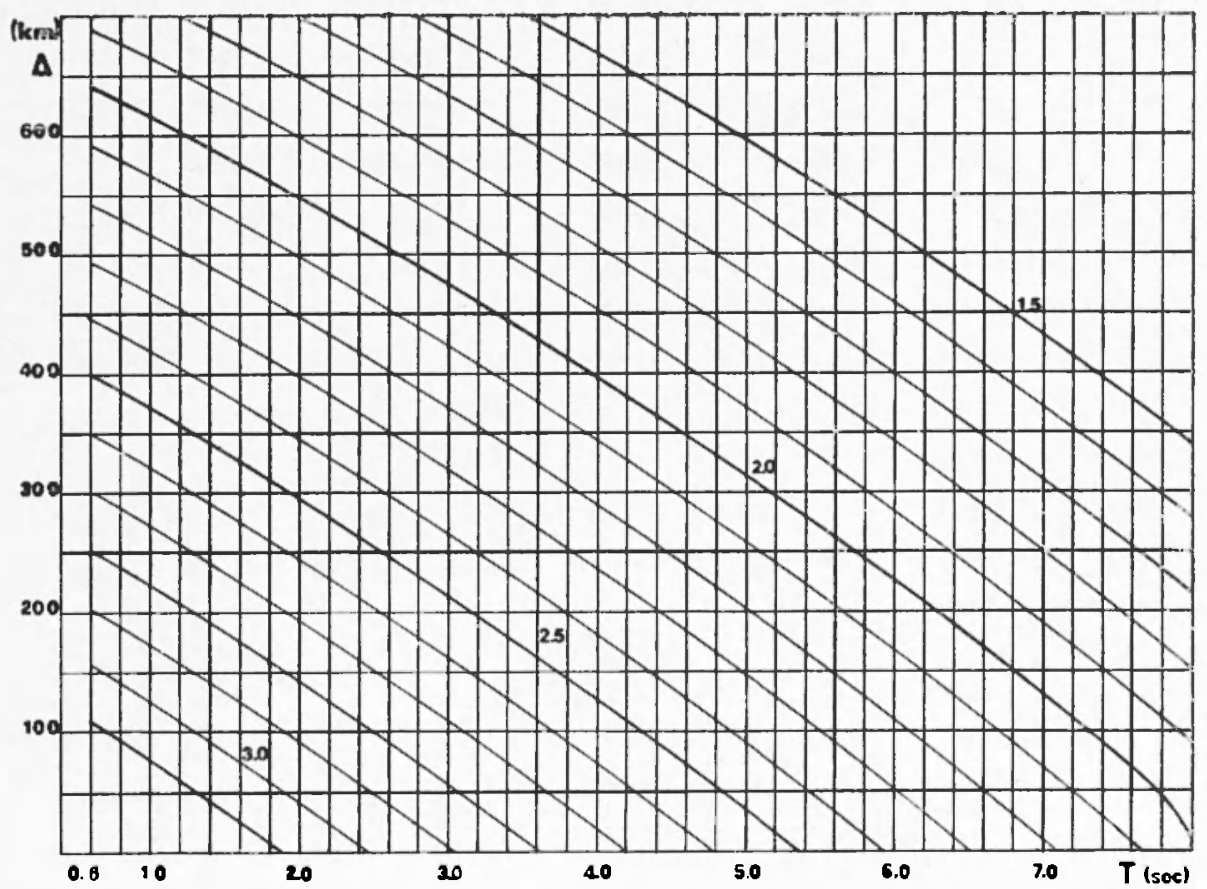

Fig. 3 - Grafici che forniscono la funzione di correzione $g(T, A)$ in funzione del periodo proprio dello strumento e della distanza epicentralc. 


\section{Tabella 2}

Funzione di correzione $g\left(T_{x}, \Delta\right)$.

\begin{tabular}{rllll}
\hline$T^{\prime}$ & $20 \mathrm{~km}$ & $130 \mathrm{~km}$ & $270 \mathrm{~km}$ & $530 \mathrm{~km}$ \\
\hline 0.8 & 3.0509 & 2.4738 & 2.5667 & 2.8307 \\
1.2 & 3.1940 & 2.5074 & 2.2829 & 2.2024 \\
1.3 & 3.0785 & 2.3157 & 2.3493 & 2.2138 \\
1.6 & 3.1335 & 2.6035 & 2.1620 & 2.0390 \\
1.8 & 3.1303 & 2.3345 & 2.2283 & 1.9804 \\
2.3 & 2.9075 & 2.4712 & 2.3168 & 1.7585 \\
2.4 & 2.9304 & 2.4933 & 2.3010 & 1.7721 \\
2.6 & 2.8997 & 2.5475 & 2.3615 & 1.6636 \\
3.9 & 2.9133 & 2.3841 & 2.1379 & 1.7987 \\
4.2 & 2.8694 & 2.5698 & 2.0510 & 2.0531 \\
4.4 & 2.7982 & 2.4136 & 2.0692 & 1.7944 \\
4.6 & 2.7332 & 2.4369 & 1.9725 & 1.9467 \\
5.0 & 2.6348 & 2.2980 & 2.1081 & 1.7492 \\
6.7 & 2.4551 & 2.2027 & 2.0627 & 1.7070 \\
10.0 & 2.3831 & 2.0865 & 1.9257 & 1.4326 \\
\hline
\end{tabular}


TABELLA 3

Magnitudo del terremoto del 23.10.1970.

\begin{tabular}{|c|c|c|c|c|c|c|c|}
\hline Stazione & $\Delta(\mathrm{km})$ & $f(\Delta)$ & n. & Comp. & $\Delta_{\max }$ & $M$ & Scarto \\
\hline Mineo (CT) & 147.3 & 3.284 & 20 & ORIZ & 27.0 & 6.041 & 0.372 \\
\hline \multirow[t]{6}{*}{ Catania } & 106.0 & 3.036 & 1 & $N E$ & 25.0 & 4.880 & -0.789 \\
\hline & & & 1 & $N W$ & 43.5 & 5.120 & --0.549 \\
\hline & & & 2 & $S$ & 39.5 & 5.593 & -0.076 \\
\hline & & & 2 & $N$ & 48.0 & 5.678 & 0.009 \\
\hline & & & 21 & $E$ & 23.5 & 6.030 & 0.361 \\
\hline & & & 21 & $N$ & 38.0 & 6.239 & 0.570 \\
\hline Carloforte (CA) & 685.4 & 5.071 & 22 & $N E$ & 0.9 & 5.016 & -0.653 \\
\hline (Is. S. Pietro) & & & 22 & $N W$ & 0.7 & 4.907 & -0.762 \\
\hline Caggiano (SA) & 286.1 & 4.516 & 23 & ORIZ & 14.5 & 6.478 & 0.809 \\
\hline Rocca di Papa & 503.7 & 4.707 & 19 & $N S$ & 31.5 & 6.004 & 0.335 \\
\hline \multirow[t]{5}{*}{ (Roma) } & & & 19 & $E W$ & 22.5 & 5.858 & 0.189 \\
\hline & & & 6 & $N W$ & 14.0 & 5.852 & 0.183 \\
\hline & & & 6 & $N E$ & 7.0 & 5.551 & -0.118 \\
\hline & & & 7 & $E W$ & 2.6 & 5.725 & 0.056 \\
\hline & & & 7 & $N E$ & 1.0 & 5.310 & -0.359 \\
\hline Ximeniano (FI) & 755.8 & 5.212 & 8 & $O R I Z$ & 3.5 & 5.295 & -0.374 \\
\hline \multirow[t]{2}{*}{ Moncalieri (TO) } & 1040.3 & 5.781 & 24 & $N S$ & 8.5 & 5.631 & -0.038 \\
\hline & & & 25 & $E W$ & 17.5 & 6.009 & 0.340 \\
\hline Padova & 889.6 & 5.479 & 10 & $O R I Z$ & 27.5 & 6.102 & 0.433 \\
\hline Venezia & 879.2 & 5.458 & 13 & $N S$ & 11.0 & 5.720 & 0.051 \\
\hline
\end{tabular}


5. APPLICAZIONE DEL METODO E CONCluSiONI.

Nella tabella 3 riportiamo i risultati di un'applicazione del metodo illustrato nei precedenti paragrafi. Essi sono stati ottenuti mediante un programma per elaboratore elettronico in cui, come dati fissi, si trovano inserite le coordinate e le caratteristiche strumentali delle varie stazioni sismiche e, come dati da variare di volta in volta, si devono fornire le coordinate dell'evento e le ampiezze rilevate sui sismogrammi delle singole stazioni.

L'esempio si riferisce al terremoto di Ferruzzano (Reggio Calabria) del 23.10.1907 alle ore 2027 ca. $(G M T)$, avente come coordinate epicentrali: $\varphi=30^{\circ} 02^{\prime} N, \lambda=16^{\circ} 05 E$.

I dati sono stati tratti dall'opera citata in bibliografia $\left(^{2}\right)$.

La magnitudo calcolata dalla media di 21 osservazioni adiversa distanza e con diversi strumenti, vale $M=5.67$, con una deviazione standard percentuale del $7.66 \%$.

Citiamo, a titolo di esempio, che per lo stesso terremoto, Karnik $\left({ }^{3}\right)$ aveva ottenuto una magnitudo pari a $M=5.9$.

In conclusione possiamo affermare che, data la semplicità di applicazione, il metodo descritto nel presente lavoro, potrù contribuire al recupero di dati ancora validi per la conoscenza dei terremoti storici.

\section{BIBLIOGRAFIA}

(1) Aurelio, V., Console, R., Gasparin1, C., 1973. - La magnitudo calcolata mediante sismografi a breve periodo. "Annali di Geofisica », 26, 4, pp. 693-703.

(2) Bollettino della Società Sismologica Italiana. 1910. 14, pp. 455-461.

(3) Karník, V., 1969. - Seismicity of the Eutropean area. Reidel Publish. Co., Holland, parte 1.

(+) Richter, C. F., 1935. - An instrumental earthquakes magnitude scale. "Bull. of the Scism. Soc. Amer. », 1. 